\title{
High endocan levels are associated with the need for mechanical ventilation among patients with severe sepsis
}

\author{
To the Editor:
}

Sepsis affects more than 750000 Americans each year, with a mortality rate close to $30 \%$ [1]. A significant amount of resources has been put into improving our understanding of sepsis and developing new therapies. A push for early sepsis recognition and subsequent timely treatment has led to an interest in inflammatory biomarkers to identify sepsis and its severity [2]. Respiratory dysfunction occurs in up to $81 \%$ of cases and a significant proportion requires ventilatory support [1]. Studies have looked at a variety of biomarkers, from inflammatory cytokines such as interleukin (IL)-6 to endothelial proteins like intercellular adhesion molecule-1, to identify patients at greatest risk of developing respiratory compromise [3].

Endocan is a $50-\mathrm{kDa}$ dermatan sulfate proteoglycan initially discovered in lung and kidney endothelial cells [4] and later in the vasculature and tumour cells [5]. Inflammatory mediators stimulate endocan expression and elevated levels have been demonstrated in septic pneumonia [6] and in community-acquired pneumonia patients [7]. Endocan also has been shown to be a marker for the severity of acute respiratory distress syndrome (ARDS)[3], which may be explained by an incompletely described pathway involving hypoxia inducible factor (HIF)-1 stimulating endocan expression [8]. Understanding the relationship between endocan and respiratory failure can help predict the severity of disease, the need for more aggressive management and the development of new therapies for management of hypoxia in various disease states. Therefore, the primary objective of our study is to determine the association between endocan and the need for mechanical ventilation secondary to sepsis-related respiratory failure during intensive care unit (ICU) admission in septic patients.

We performed a secondary analysis of a prospective cohort of patients admitted with severe sepsis at two academic institutions, the South Texas Veterans Health Care System and the University Hospital in San Antonio, TX, from November 2007 to October 2012 [9]. Blood samples to measure endocan levels were drawn from all patients within $48 \mathrm{~h}$ of developing severe sepsis and prior to needing mechanical ventilation. All patients received guidelines-based therapy, including antibiotics during their hospital stay. The study was approved by the local Institutional Review Board (clinicaltrials.gov identifier NCT00708799) [9]. Patients met the following inclusion criteria: age $\geqslant 18$ years, not requiring mechanical ventilation during the first $48 \mathrm{~h}$ of ICU admission, suspected or documented infection with systemic inflammatory reaction syndrome (SIRS) [10], and development of organ dysfunction. Patients were excluded if they met any of the following: 1) receiving current macrolide antibiotic therapy; 2) immunosuppressed [9].

Patients were followed daily during their ICU stay and evaluated for their requirement of mechanical ventilation and for 1 year after hospital discharge for survival. Mechanical ventilation was defined as either noninvasive or invasive forms of assisting or replacing spontaneous breathing. The Acute Physiology and Chronic Health Evaluation II (APACHE II) score [11] and the Sequential Organ Failure Assessment (SOFA) score [12] were calculated on admission to the ICU. Within $48 \mathrm{~h}$ of onset of severe sepsis and admission to the ICU, a blood sample was collected from each patient and detection of endocan levels was determined using a commercially available ELISA kit. The primary outcome was receipt of mechanical ventilation during ICU admission and the secondary outcome was ICU mortality.

@ERSpublications

Endocan may have a role as a predictive biomarker for need for mechanical ventilation in patients with severe sepsis http://ow.ly/raKd30bSqTk

Cite this article as: Mangat M, Amalakuhan B, Habib S, et al. High endocan levels are associated with the need for mechanical ventilation among patients with severe sepsis. Eur Respir J 2017; 50: 1700013 [https:// doi.org/10.1183/13993003.00013-2017]. 
Values are expressed as median with interquartile range. Fisher's exact test was used to compare categorical values between groups. The Mann-Whitney U-test was used for nonparametric and Student's t-test analysis of continuous variables when appropriate. A p-value $<0.05$ was deemed statistically significant. A receiver operating characteristic (ROC) curve was used to assess the discrimination capacity of endocan to predict the requirement of mechanical ventilation.

31 patients met the study criteria, with eight patients needing mechanical ventilation during the ICU hospitalisation. Initiation of mechanical ventilation in our cohort was within $72 \mathrm{~h}$ of the endocan level being drawn. Table 1 shows the characteristics of the patients by whether they received mechanical ventilation or not. The primary sources of infection in both groups were pulmonary and urinary tract. The patients requiring ventilatory support had a trend towards a higher SOFA score.

Patients with severe sepsis requiring mechanical ventilation during ICU admission had significantly higher endocan levels than those not requiring mechanical ventilation. The median endocan levels were 2.39 (IQR (interquartile range) $1.7-2.7$ ) $\mathrm{ng} \cdot \mathrm{mL}^{-1}$ and 4.86 (IQR 3.2-9.8) $\mathrm{ng} \cdot \mathrm{mL}^{-1}$ in patients not requiring mechanical ventilation and requiring mechanical ventilation, respectively $(p \leqslant 0.001)$. Thus, levels of endocan above $4.86 \mathrm{ng} \cdot \mathrm{mL}^{-1}$ at the onset of severe sepsis were predictive of the later need for mechanical ventilation. The area under the ROC curve (AUC) for the need for mechanical ventilation was 0.89 . No statistically significant differences in endocan levels were noted for ICU mortality (median (IQR) 4.9 (1.812.6) versus $\left.2.5(1.9-3.0) \mathrm{ng} \cdot \mathrm{mL}^{-1} ; \mathrm{p}=0.2\right)$.

\section{TABLE 1 Baseline demographics and clinical characteristics of patients at randomisation}

\begin{tabular}{|c|c|c|c|}
\hline Variables & No ventilatory support & Ventilatory support & $\mathrm{p}$-value \\
\hline Patients n & 23 & 8 & \\
\hline Age years & $59(51-65)$ & $58(45-79)$ & 0.6 \\
\hline Male & $19(83)$ & 7 (87) & 0.6 \\
\hline \multicolumn{4}{|l|}{ Comorbid conditions } \\
\hline Obesity & $7(30)$ & 3 (37) & 0.5 \\
\hline Prior cancer & $7(30)$ & 1 (13) & 0.8 \\
\hline Cardiovascular disease & $4(17)$ & $2(25)$ & 0.4 \\
\hline Chronic heart failure & $1(4)$ & $1(13)$ & 0.4 \\
\hline COPD & $3(13)$ & $1(13)$ & 0.7 \\
\hline Chronic kidney disease & $1(4)$ & 1 (13) & 0.4 \\
\hline Depression & 3 (13) & $0(0)$ & 0.3 \\
\hline Diabetes mellitus & $12(52)$ & 3 (37) & 0.3 \\
\hline Hyperlipidaemia & $4(17)$ & $1(13)$ & 0.6 \\
\hline Liver disease & $1(4)$ & $0(0)$ & 0.7 \\
\hline Tobacco use & 7 (30) & $2(25)$ & 0.5 \\
\hline Alcohol use & $6(26)$ & $0(0)$ & 0.2 \\
\hline Asthma & $0(0)$ & $1(12)$ & 0.2 \\
\hline \multicolumn{4}{|l|}{ Source of infection } \\
\hline Pulmonary & $4(17)$ & 3 (37) & 0.5 \\
\hline Urinary tract & $13(56)$ & $2(25)$ & 0.3 \\
\hline $\mathrm{Gl}$ & $1(4)$ & 3 (37) & 0.4 \\
\hline Skin & $4(17)$ & $0(0)$ & 0.3 \\
\hline Endocarditis & $1(4)$ & $0(0)$ & 0.9 \\
\hline \multicolumn{4}{|l|}{ Severity of the disease } \\
\hline SOFA & $6.0 \pm 2.8$ & $8.6 \pm 3.6$ & 0.06 \\
\hline Number of organ failure & $1(1-2)$ & $2.5(1-3)$ & 0.08 \\
\hline $\mathrm{PO}_{2} \mathrm{mmHg}$ & $81.7 \pm 21.9$ & $75.4 \pm 26$ & 0.5 \\
\hline $\mathrm{PaO}_{2} / \mathrm{FiO}_{2} \mathrm{mmHg}$ & $252.6 \pm 120.2$ & $219.1 \pm 131.7$ & 0.6 \\
\hline $\mathrm{PCO}_{2} \mathrm{mmHg}$ & $32.8 \pm 7.2$ & $34.9 \pm 11.1$ & 0.5 \\
\hline Heart rate $\min ^{-1}$ & $103 \pm 22$ & $119 \pm 19$ & 0.08 \\
\hline Respiratory rate $\min ^{-1}$ & $28 \pm 6.7$ & $32 \pm 8.6$ & 0.2 \\
\hline Systolic blood pressure $\mathrm{mmHg}$ & $89 \pm 9.8$ & $91 \pm 26$ & 0.7 \\
\hline Temperature $\mathrm{C}$ & $37.3 \pm 1.4$ & $37.0 \pm 2.2$ & 0.6 \\
\hline
\end{tabular}

Data are presented as median (interquartile range), $\mathrm{n}(\%)$ or mean $\pm \mathrm{SD}$, unless otherwise indicated. $\mathrm{GI}$ : gastrointestinal; SOFA: sequential organ failure assessment; COPD: chronic obstructive pulmonary disease; $\mathrm{FiO}_{2}$ : inspiratory oxygen fraction; $\mathrm{PaO}_{2}$ : arterial oxygen tension; $P_{2}$ : partial pressure of oxygen; $P_{\mathrm{CO}_{2}}$ : partial pressure of carbon dioxide. 
Endocan release is stimulated by multiple pathways, including endothelial injury and during sepsis by pro-inflammatory cytokines. BECHARD et al. [13] demonstrated higher endocan levels in patients with septic shock than in healthy controls. In vivo, higher endocan levels are associated with sepsis severity and multiple organ failure, as demonstrated in the works by SCHERPEREel et al. [6] and MinajLovic et al. [14].

Another pathway of interest is the relationship between endocan and HIF-1. In a colorectal cancer cell model, hypoxia induces HIF-1 expression with subsequent endocan production [8]. Given the presence of endocan and HIF-1 in the lungs, it would be reasonable to deduce that this pathway also may be present in normal noncancerous human tissue cells as well. Our finding of increased endocan levels in those patients requiring mechanical ventilation may be explained by subclinical hypoxia at the cellular level resulting in HIF-1 production. The degree of endocan elevation may then be used as a predictor of disease severity and the development of respiratory failure requiring mechanical ventilation. Previous studies have shown contradictory associations between endocan and respiratory failure. One study in fact demonstrated that lower endocan levels were associated with respiratory failure in a group of 20 patients with septic shock [15]. Another study by TANG et al. [3] showed that higher endocan levels were predictive of further decline in respiratory status in those with ARDS.

In terms of the association between endocan levels and mortality, limited data are available. In the study by TANG et al. [3], elevated endocan levels were associated with higher mortality rates only in a cohort of patients with ARDS. Although our study did not find an association between endocan levels and mortality in patients with respiratory failure as defined by those requiring mechanical ventilation, our patients did not meet the diagnostic criteria for ARDS and had less severe forms of respiratory failure.

Our study has some limitations that should be acknowledged. First, our sample size was small based upon strict inclusion/exclusion criteria. Second, endocan levels were obtained only on ICU admission, limiting our ability to assess dynamic changes in serum levels throughout the patient's clinical course, including response to treatment. Furthermore, the mechanically ventilated group had a trend towards greater severity of illness, which may potentially affect the interpretation of the serum endocan levels. Lastly, our results require further validation in other patient cohorts to confirm the strength of the association between endocan levels and its potential predictive capacity for the need for mechanical ventilation.

In conclusion, there is evidence that endocan may have a role as a predictive biomarker for the need for mechanical ventilation in patients with severe sepsis. High endocan levels were not associated with mortality. The strength of these associations will need to be further validated in a larger cohort of patients.

Mandeep Mangat $^{1}$, Bravein Amalakuhan ${ }^{1}$, Sheila Habib ${ }^{1}$, Luis F. Reyes ${ }^{1,2}$, Cecilia A. Hinojosa ${ }^{1}$, Alejandro H. Rodriguez ${ }^{3}$, Nilam J. Soni ${ }^{1,2}$, Antonio Anzueto ${ }^{1,2}$, Stephanie M. Levine ${ }^{1,2}$, Jay I. Peters ${ }^{1,2}$, Stefano Aliberti $\oplus^{4}$, Oriol Sibila ${ }^{5}$, Jordi Rello ${ }^{6}$, Martin Witzenrath ${ }^{7}$, Grant W. Waterer ${ }^{8}$, Ignacio Martin-Loeches ${ }^{9}$, Jose Blanquer ${ }^{10}$, Francisco Sanz ${ }^{11}$, Pedro J. Marcos $\mathbb{1}^{12}$, Jordi Solé-Violán ${ }^{13}$, James D. Chalmers ${ }^{14}$, Charles Feldman ${ }^{15}$, Richard G. Wunderink ${ }^{16}$, Charles S. Dela Cruz ${ }^{17}$, Carlos J. Orihuela ${ }^{18}$ and Marcos I. Restrepo ${ }^{1,2}$

${ }^{1}$ University of Texas Health Science Center San Antonio, San Antonio, TX, USA. ${ }^{2}$ South Texas Veterans Health Care System, San Antonio, TX, USA. ${ }^{3}$ Critical Care Department, Joan XXIII University Hospital and Pere Virgili Health Institute, CIBERES, Tarragona, Spain. ${ }^{4}$ University of Milan Bicocca, Clinica Pneumologica, Monza, Italy. ${ }^{5}$ Servei de Pneumologia, Departament de Medicina, Hospital Santa Creu i Sant Pau, Universitat Autònoma de Barcelona, Spain. ${ }^{6}$ Critical Care Department, Hospital Universitario Vall d'Hebron, CIBERES, Barcelona, Spain. ${ }^{7}$ Department of Infectious Diseases and Pulmonary Medicine, Charité-Universitätsmedizin Berlin and SFB-TR84 "Innate Immunity of the Lung", Berlin, Germany. ${ }^{8}$ Royal Perth Hospital Unit, University of Western Australia, Perth, Australia. ${ }^{9}$ St. James's Hospital, Trinity Centre for Health Sciences, CIBERES, Dublin, Ireland. ${ }^{10}$ Unidad Cuidados Intensivos Respiratorios, Hospital Clínic Universitari, Valencia, Spain. ${ }^{11}$ Pulmonology Department, Consorci Hospital General Universitari de Valencia, Valencia, Spain. ${ }^{12}$ Servicio de Neumología, Instituto de investigación Biomédica de A Coruña (INIBIC), Complejo Hospitalario Universitario de A Coruña (CHUAC), Sergas, Universidade da Coruña (UDC), A Coruña, Spain.

${ }^{13}$ Intensive Care Unit, Hospital Universitario Dr. Negrín, CIBERES, Las Palmas, Gran Canaria, Spain. ${ }^{14}$ University of Dundee, Dundee, UK. ${ }^{15}$ Department of Internal Medicine, Charlotte Maxeke Johannesburg Academic Hospital, Johannesburg, South Africa, Faculty of Health Sciences, University of the Witwatersrand, Johannesburg, South Africa. ${ }^{16}$ Division of Pulmonary and Critical Care Medicine, Department of Medicine, Feinberg School of Medicine, Northwestern University, Chicago, IL, USA. ${ }^{17}$ Division of Pulmonary and Critical Care Medicine, Yale University, New Haven, CT, USA. ${ }^{18}$ Department of Microbiology, The University of Alabama at Birmingham, Birmingham, AL, USA.

Correspondence: Marcos I. Restrepo, South Texas Veterans Health Care System ALMD, 7400 Merton Minter Boulevard, San Antonio, TX 78229, USA. E-mail: restrepom@uthscsa.edu

Received: Jan 032017 | Accepted after revision: March 272017

Conflict of interest: Disclosures can be found alongside this article at erj.ersjournals.com

\section{References}

1 Angus DC, Linde-Zwirble WT, Lidicker J, et al. Epidemiology of severe sepsis in the United States: analysis of incidence, outcome, and associated costs of care. Crit Care Med 2001; 29: 1303-1310. 
Pierrakos C, Vincent JL. Sepsis biomarkers: a review. Crit Care 2010; 14: R15.

Tang L, Zhao Y, Wang D, et al. Endocan levels in peripheral blood predict outcomes of acute respiratory distress syndrome. Mediators Inflamm 2014; 2014: 625180.

4 Lassalle P, Molet S, Janin A, et al. ESM-1 is a novel human endothelial cell-specific molecule expressed in lung and regulated by cytokines. J Biol Chem 1996; 271: 20458-20464.

5 Aitkenhead M, Wang SJ, Nakatsu MN, et al. Identification of endothelial cell genes expressed in an in vitro model of angiogenesis: induction of ESM-1, (beta)ig-h3, and NrCAM. Microvasc Res 2002; 63: 159-171.

6 Scherpereel A, Depontieu F, Grigoriu B, et al. Endocan, a new endothelial marker in human sepsis. Crit Care Med 2006; 34: 532-537.

7 Kao SJ, Chuang CY, Tang CH, et al. Plasma endothelial cell-specific molecule-1 (ESM-1) in management of community-acquired pneumonia. Clin Chem Lab Med 2014; 52: 445-451.

8 Kim JH, Park MY, Kim CN, et al. Expression of endothelial cell-specific molecule-1 regulated by hypoxia inducible factor-1alpha in human colon carcinoma: impact of ESM-1 on prognosis and its correlation with clinicopathological features. Oncol Rep 2012; 28: 1701-1708.

9 Amalakuhan B, Habib SA, Mangat M, et al. Endothelial adhesion molecules and multiple organ failure in patients with severe sepsis. Cytokine 2016; 88: 267-273.

10 Dellinger RP, Levy MM, Rhodes A, et al. Surviving sepsis campaign: international guidelines for management of severe sepsis and septic shock: 2012. Crit Care Med 2013; 41: 580-637.

11 Knaus WA, Draper EA, Wagner DP, et al. APACHE II: a severity of disease classification system. Crit Care Med 1985; 13: 818-829.

12 Vincent JL, Moreno R, Takala J, et al. The SOFA (Sepsis-related Organ Failure Assessment) score to describe organ dysfunction/failure. Intensive Care Med 1996; 22: 707-710.

13 Bechard D, Meignin V, Scherpereel A, et al. Characterization of the secreted form of endothelial-cell-specific molecule 1 by specific monoclonal antibodies. J Vasc Res 2000; 37: 417-425.

14 Mihajlovic DM, Lendak DF, Brkic SV, et al. Endocan is useful biomarker of survival and severity in sepsis. Microvasc Res 2014; 93: 92-97.

15 Palud A, Parmentier-Decrucq E, Pastre J, et al. Evaluation of endothelial biomarkers as predictors of organ failures in septic shock patients. Cytokine 2015; 73: 213-218. 